\title{
EXTRACTION AND ANALYSIS OF TRADITIONAL CHINESE MEDICINE CROPS BASED ON MULTITEMPORAL HIGH RESOLUTION DATA-TAKING QIAOCHENG DISTRICT OF BOZHOU AS AN EXAMPLE
}

\author{
Hong $\mathrm{Yu}^{1}$, Jianjun $\mathrm{He}^{1}{ }^{1}$, Huizhen Zhou ${ }^{1,2}$, Feng Guan $^{1}$,Longlong $\mathrm{Li}^{1}{ }^{1}$ Baiyang Ren ${ }^{1}$, Zhiyong Wang ${ }^{3}$ \\ ${ }^{1}$ Twenty First Century Aerospace Technology Co., Ltd., Beijing, China - (yuhong, hejj, zhouhz, guanfeng, lill,renby) @ 21at.com.cn \\ ${ }^{2}$ State Key Laboratory of Earth Surface Processes and Resource Ecology, School of Natural Resources, Faculty of Geographical \\ Science, Beijng Normal University, Beijing, China \\ ${ }^{3}$ Beijing Engineering Research Center of Small Satellite Remote Sensing Information, Beijing, China - wangzy@ 21at.com.cn
}

KEY WORDS: Multitemporal, Remote,SensingData, Chinese Medicine Crops, Interpretation, Information Extraction

\begin{abstract}
:
Remote sensing technology has become an important method to rapidly acquireing of planting layout and composition of regional crops.It is very important to accurately master the planting area of Chinese medicine crops in the Characteristic planting area because it relations to accurately master the cultivation of Chinese medicine crops, formulate related policies and adjustment of crop planting structure. The author puts forward a method of using remote sencing technology for momitoring Chinese medicine which has good applicability and generalization. This paper took Qiaocheng District of Bozhou as an example to Verify the feasibility of the method,providing a reference for solving the problem of interpretation and extraction of Chinese medicinal materials in the region.
\end{abstract}

\section{INSTRUCTION}

With the development of China's economy and the improvement of people's living standard, the demand for Chinese medicine crops is increasing. According to the statistics, in 2015, the planting area of Chinese medicine crops in China was about 504.55 million mu, and the production of Chinese medicine crops was about 3638,000 tons. Comparing with the same period of last year, the production increased by $3.35 \%$ and showed an increasing trend year by year. In the characteristic regions of Chinese medicine crops, the cultivation of Chinese medicine crops is not only related to the adjustment of local agricultural planting structure, but also an important content of the development of efficient ecological agriculture. It is even an effective means for farmers to increase their income. Therefore, it is necessary for the government departments to guide the agricultural work and formulate relevant agricultural policies in a timely and accurately manner.(Chen et al., 2005; Zhou et al., 2004)

Traditional Chinese medicine resource investigation method can't meet the demand of modern statistical investigation any longer(Guo et al., 2005; Chen et al., 2005). Because traditional method usually needs long cycle and large workload, relying on artificial estimation and acquisition quantity estimation, lack of scientific nature and it cannot resolve the spatial distribution and other issues. As a new and comprehensive technology, remote sensing technology has the advantages of macroscopic, rapid and dynamic, and it can achieve large area synchronized observation, so this method has become an important means of getting the area's planted crop layout in a short time.

Although Modis and Landsat data are still dominant a station, but as the launch of domestic satellites, the domestic satellites such as High-resolution series satellites are more and more used as the data source. Recently, domestic and foreign experts and scholars have made some progress in the research on the cultivation and monitoring of Chinese medicine crops by means of remote sensing. For example, Sun Yuzhang et al. proposed the monitoring of wild atractylodes resources method, which is a pioneering and demonstration of research on wild medicinal plants using remote sensing technology in China(Sun et al., 2007). Pan Huijiao et al. take the Tibetan medicine Herpetospermum pedunculosum Baill cultivation as an example in Luding county. They used remote sensing and GIS technology, taking TM and ETM images as information sources and combined field survey data to extract the Tibetan medicine's planting area. This technique shows that remote sensing can be used to monitor the planting area of Chinese medicinal crops in complex mountainous environment(Pan et al., 2014). Zhang Xiaobo et al. discussed the principle, method and standard of using remote sensing to monitor the cultivation of Chinese medicinal crops(Zhang et al., 2009 ). In general, most researchers on Chinese medicine used low resolution image to extract planting information, leading to a result that it is easy to ignore the influence from other crops using a lowresolution data during this period. At the same time, in the process of information extraction, supervision classification and artificial visual interpretation are the usual methods we adopted. But these methods are too simple, and it is a large workload when facing a large-scale application, and the accuracy on using this extraction method is relatively single, the accuracy is relatively low.

This paper takes Qiaocheng district, Bozhou city, Anhui province as the experimental area, based on the Chinese independent research and development production Highresolution data, using object-oriented technology, building the characteristics of spectral, texture, normalized vegetation index, brightness and other characteristic pattern sets and combining with the survey data of GPS ground sample to monitor the planting area of Chinese medicine crops in Qiaocheng District. This method has made a new breach in the extraction of regional characteristic crop information.In this paper, On the

\footnotetext{
* Corresponding author
} 
basis of extraction results, the results of information extraction are verified and analysed.The extracted planting area of Chinese medicine crops in Qiaocheng district is $655.3 \mathrm{~km}^{2}$, and the extraction accuracy is $93.56 \%$. The results show that the technical method in this paper has a good applicability and generalization, and it can provide a reference and a solution for the information interpretation and extraction work of Chinese medicine crops in more areas.

\section{DATA SOURCES AND RESEARCH METHODS}

With the development of China's economy and the improvement of people's living standard, the demand for Chinese medicine crops is increasing. According to the statistics, in 2015, the planting area of Chinese medicine crops in China was about 504.55 million mu, and the production of Chinese medicine crops was about 3638,000 tons. Comparing with the same period of last year, the production increased by $3.35 \%$ and showed an increasing trend year by year. In the characteristic regions of Chinese medicine crops, the cultivation of Chinese medicine crops is not only related to the adjustment of local agricultural planting structure, but also an important content of the development of efficient ecological agriculture. It is even an effective means for farmers to increase their income. Therefore, it is necessary for the government departments to guide the agricultural work and formulate relevant agricultural policies in a timely and accurately manner.

\subsection{Study Area}

The study area is located in Qiaocheng District, Bozhou City, Anhui Province (as shown in Figure 1), which is located in the northwest of Anhui province, the area from $115^{\circ} 33^{\prime}$ to $116^{\circ}$ $06^{\prime}$ east longitude and $33^{\circ} 25^{\prime}$ to $34^{\circ} 05^{\prime}$ north latitude, 36 meters on average. The region belongs to the warm temperate semi-humid monsoon climate zone. The annual average temperature is $14.5^{\circ} \mathrm{C}$ and the annual average rainfall is $805 \mathrm{~mm}$. This area is rich in light, proper temperature and water resources, which is suitable for all kinds of crops.Qiaocheng city has been known as the "Chinese medicine capital" since ancient times, which is the first of the four major drugs in the country, with a long history of planting traditionalChinese medicine crops, and nearly 300 species of traditional Chinese medicine crops were cultivated.

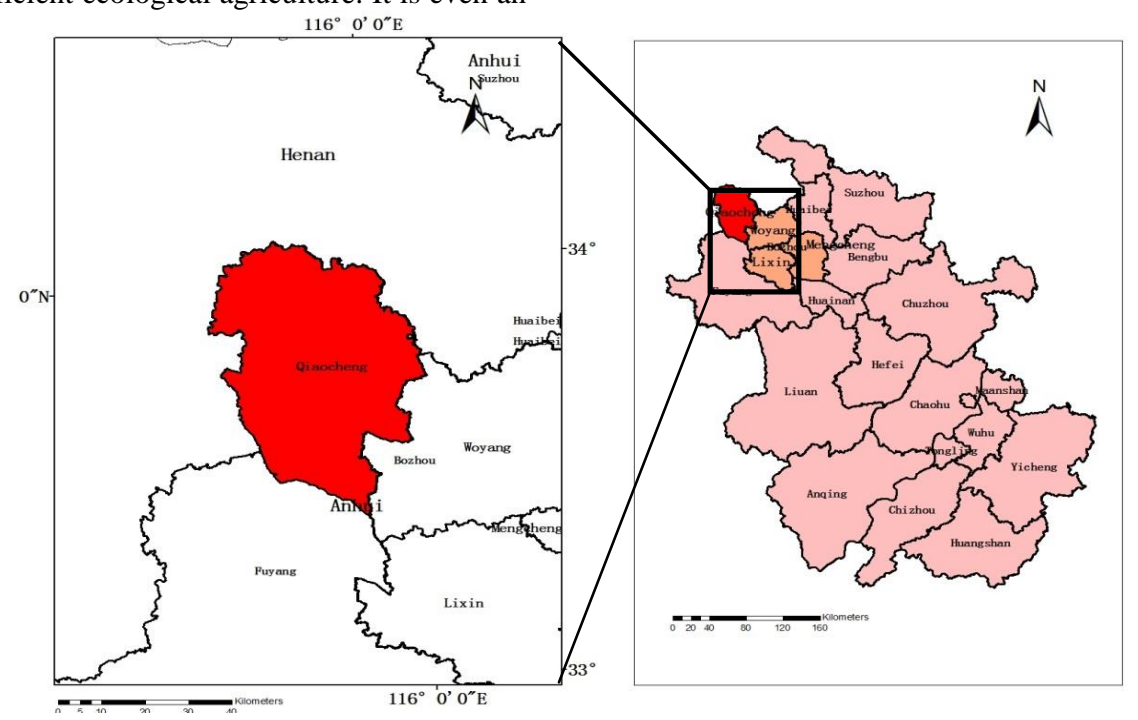

Figure 1.The geographical location of the study area

\subsection{Data source and pretreatment}

This study used High-resolution data(GF-1) as the experimental data,while the data satisfying wide coverage and high resolution. GF - 1 satellite data is the first satellite of High resolution to earth observation system which is national science and technology major special projects. The satellite equipped with two cameras which is $2 \mathrm{~m}$ resolution by full-color and $8 \mathrm{~m}$ resolution multispectral (PMS),also carrying four $16 \mathrm{~m}$ resolution multispectral (WFV) cameras. The main parameters of GF - 1 satellite is shown in Table 1.

The data used in this study included three GF-1 images and the resolution is $16 \mathrm{~m}$. The data covered the key growth period of traditionalChinese medicine crops in 2016, as shown in Table 2. The three images are both good of definition and less in cloud, which is beneficial to identification of Chinese medicine crops in the critical period of growth.

In order to maximize the accuracy of information extraction, the images were pretreated with orthophoto correction, geometric correction, uniform color, and administrative boundary vector cutting. For example, the historical SPOT images of the experimental area were as a reference image for geometric correction.As the quadratic polynomial method, each image choosing between 20 and 30 control points to correct and the error controlled within 1 pixel.The preprocessing results of GF1 images in the research area are shown in Figure 2.

\begin{tabular}{cccc}
\hline Parameters & & The cameraof WFV & The cameraof PMS \\
\hline Spectrum rang & full-color & & $0.45-0.90 \mathrm{um}$ \\
\cline { 2 - 4 } & & $0.45-0.52 \mathrm{um}$ & $0.45-0.52 \mathrm{um}$ \\
& multispectral & $0.52-0.59 \mathrm{um}$ & $0.52-0.59 \mathrm{um}$ \\
& & $0.63-0.69 \mathrm{um}$ & $0.63-0.69 \mathrm{um}$
\end{tabular}


The International Archives of the Photogrammetry, Remote Sensing and Spatial Information Sciences, Volume XLII-3, 2018 ISPRS TC III Mid-term Symposium "Developments, Technologies and Applications in Remote Sensing", 7-10 May, Beijing, China

\begin{tabular}{|c|c|c|c|}
\hline \multirow{3}{*}{$\begin{array}{l}\text { Spatial } \\
\text { resolution }\end{array}$} & & $0.77-0.89 \mathrm{um}$ & $0.77-0.89 \mathrm{um}$ \\
\hline & $\begin{array}{c}\text { full-color } \\
\text { multispectral }\end{array}$ & $16 \mathrm{~m}$ & $2 \mathrm{~m}$ \\
\hline & & & $8 \mathrm{~m}$ \\
\hline Breadth & & $\begin{array}{c}800 \mathrm{~km} \text { (four cameras } \\
\text { combinations) }\end{array}$ & $\begin{array}{c}\text { 60km(two cameras } \\
\text { combinations) }\end{array}$ \\
\hline $\begin{array}{l}\text { Revisit period } \\
\text { (side-sway) }\end{array}$ & & & 4days \\
\hline $\begin{array}{l}\text { Coverage } \\
\text { period (no side- } \\
\text { sway ) }\end{array}$ & & 4days & 41days \\
\hline
\end{tabular}

Table 1. Main parameters of GF - 1

\begin{tabular}{ccc}
\hline Date (month/day/year) & Sensor & Coverage \\
\hline $3 / 24 / 2016$ & WFV3 & full coverage \\
$5 / 16 / 2016$ & WFV3 & full coverage \\
$6 / 18 / 2016$ & WFV1 & full coverage \\
\hline
\end{tabular}

Table2. List of GF-1 data obtained in the experimental area

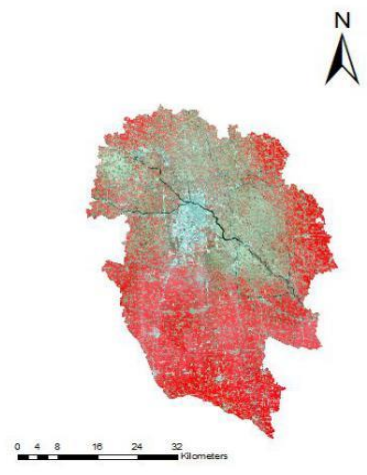

a.20160324

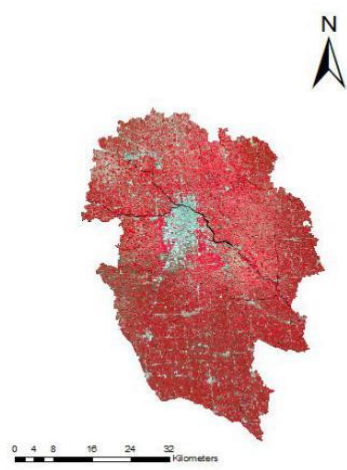

b. 20160516

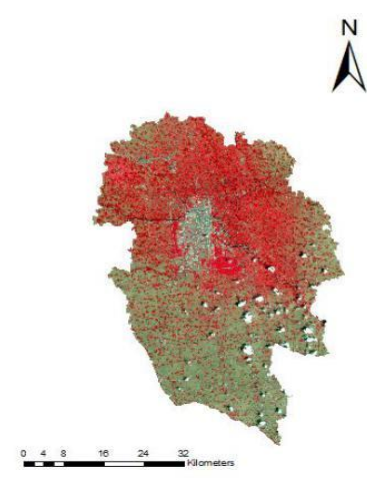

c. 20160618

Figure 2 Preprocessing results of remote sensing images in the research area

\subsection{Research methods}

Based on the GF-1images which were covering Qiaocheng District, this study interpreted traditionalChinese medicine crops in its critical growing period and combined the ground investigation data to determine the information interpretation signs for traditionalChinese medicine crops.Based on this, an object-oriented idea was applied to extract information of
traditionalChinese medicine crops in Qiaocheng District. In order to ensure the accuracy of information extraction, the scale of image segmentation and the combination of the optimal extraction features and the optimal extraction values are studied in the extraction process. The method achieved good results and the overall technical roadmap is shown in Figure 3. 


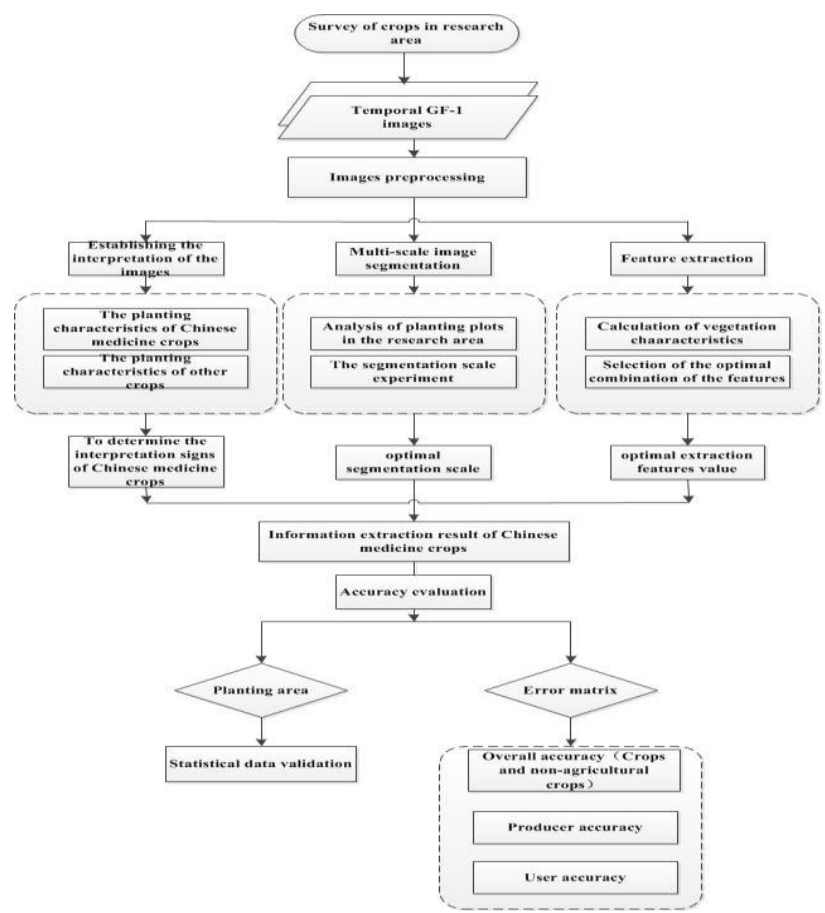

Figure 3 .The overall technical roadmap

2.3.1 Interpretation of remote sensing images: According to the research data, the area of traditional Chinese medicine crops in Qiaocheng District is mainly in the north. There are more than a dozen varieties planted in the area such as peony, radix salviae miltiorrhizae, scrophularia ningpoensis, platycodon grandiflorum, bighead atractylodes rhizome.The traditional Chinese medicine crops in Qiaocheng District is usually planted in the autumn of the first year and it reaches a period of vigorous growth around June in a second year, then began to harvest and the harvest is finished in the autumn and winter. In the Qiaocheng District, the main planting of crops are traditional Chinese medicine crops and winter wheat. When the information in the critical growth period is used to interpret the traditional Chinese medicine crops information, it is easily affected by the winter wheat information.In view of the complexity of the varieties of traditional Chinese medicine crops such as the uncertainty of harvest time, and the interference of winter wheat during the vigorous growth period, this study selected three periods when the growth of traditional Chinese medicine crops were not vigorous(the jointing stage of winter wheat), minor vigorous (the mature stage of winter wheat) and vigorous period (the harvest stage of winter wheat) to eliminate the interference of winter wheat and determine the information interpretation signs for traditional Chinese medicine crops.As shown in Table 3, there are different interpretation signs of traditional Chinese medicine crops and winter wheat in three critical growing periods.

\begin{tabular}{|c|c|c|c|}
\hline Image types & Image phase & Traditional Chinese medicine crops & Winter wheat \\
\hline \multirow{3}{*}{$\begin{array}{l}\text { GF-1 Remote } \\
\text { sening imges }\end{array}$} & $3 / 24 / 2016$ & & \\
\hline & $5 / 16 / 2016$ & & \\
\hline & $6 / 18 / 2016$ & & \\
\hline
\end{tabular}

Table3. Different interpretation signs of traditional Chinese medicine crops and winter wheat in three critical growing periods

In the image on March 24, 2016, Chinese medicine crops still exhibited near-naked information, while the winter wheat was in a period of vigorous growth and showed bright red in false color images. In the image on May 16, 2016, Chinese medicine crops were in a more vigorous growth period, presenting light red in the false color image and winter wheat showed dark red which was ripe and ready to harvest at the same time.In the image of June 18, 2016, Chinese medicine crops were in a period of vigorous growth, showing bright red color in false color images, while winter wheat had been harvested and presented with bare information. This study combines interpretation signs of different phase,eliminating winter wheat 
information interference,considering the difficulty of extraction of Chinese medicine crops and selecting the image on June 18, 2016 for the extraction of traditional Chinese medicine which was less interference information and obvious information of Chinese medicine crops.

2.3.2Multi-scale image segmentation:Multiresolution segmentation algorithm realizes image segmentation by consolidating neighboring pixels or small object segmentation through the limitation of constraint conditions, while ensuring minimum average heterogeneity between object and maximum homogeneity among objects. The multi-scale image segmentation method can fully exploit the spectral characteristics and spatial characteristics.But the segmentation scale has great influence on information extraction accuracy.If the segmentation scale is too large, it is easy to appear that one object contains multiple features. If the segmentation scale is too small, it is easy to appear that one object contains multiple features and the same ground object is divided into multiple image spots, which is not conducive to classification and information extraction. Therefore, the selection of segmentation scales is very important.

In this study, the effects of different segmentation scales on image segmentation were studied in order to select the optimal segmentation scale based on factors such as planting number, plant fragmentation degree, and the shape of the plot.

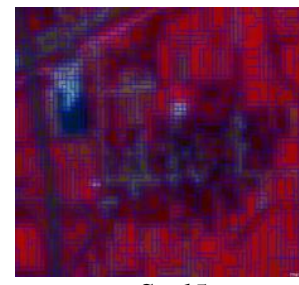

a.Scal5

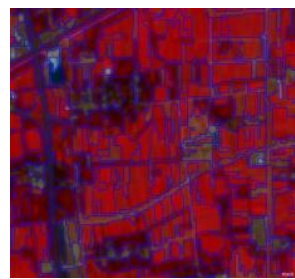

d.Scal20

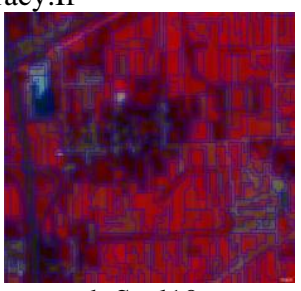

b.Scal10

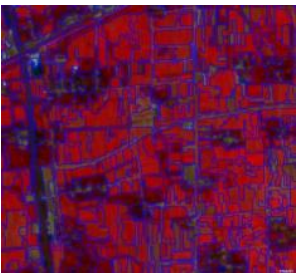

e.Scal25

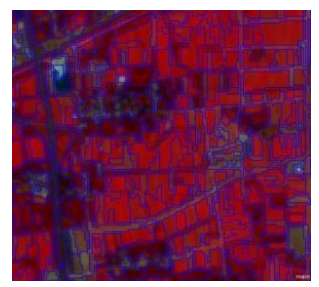

c.Scal15

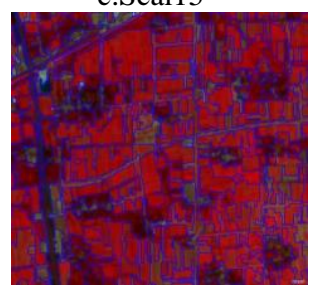

f.Scal30

Figure 4. Segmentation results of different scales

2.3.3Multi-scale image segmentation: The GF-1 remote sensin -g images of $16 \mathrm{~m}$ resolution contain rich information such as spectral, texture and others. In the information extraction process of traditional Chinese medicine crops, the classification rule set was established according to the crop growth and planting conditions in the research area.

To calculate NDVI of Chinese herbal medicine, interference of vegetation, trees (normalized vegetation index value), Brightness value (Brightness), extraction of traditional Chinese medicine information. It also extracts the Length/Width of the Chinese medicinal materials and the road, and removes the influence of the road with obvious vegetation characteristics on the information extraction results.In the process of extracting Chinese medicine crops information, NDVI (normalized vegetation index value) and Brightness (brightness value) of Chinese medicine crops, disturbance vegetation, and trees are calculated.Also the Length/Width (texture characteristic values) of Chinese medicine crops and roads are calculated. Excluding the influence of roads with obvious vegetation characteristics, the final and accurate information extraction results of Chinese medicine crops were obtained.

The normalized vegetation index NDVI can accurately reflect the phenophase changes of vegetation crops and is used for vegetation monitoring, classification, and phenological analysis. In the period of vigorous growth of Chinese medicine crops, there are obvious differences between the medicine crops and other vegetation. NDVI is calculated as follow:

$$
N D V I=\frac{\rho_{N H K}-\rho_{R}}{\rho_{W S R}+\rho_{R}}
$$

In the formula(1), $\rho_{\mathrm{NIR}}$ and $\rho_{\mathrm{R}}$ represent the reflectance in the near-infrared band and the reflectivity in the red band.
At the same time, Chinese medicine crops have higher brightness than other vegetations, so these crops can be extracted using NDVI and brightness characteristics. Brightness is calculated as follow:

$$
\bar{c}(S)=\frac{1}{W^{3}} \sum_{k=1}^{K} w_{k}^{B} \bar{c}_{k}(S)
$$

In the formula(2), $\bar{c}_{k}(S)$ represents the spectral mean of the object $\mathrm{S}$ of the $\mathrm{K}$ band, $w_{k}^{B}$ represents the weight of the $\mathrm{K}$ band, $W^{B}$ represents the sum of weight of each band.

Due to the fragmentation of some Chinese medicine planting plots, it is easy to classify roads with vegetation as Chinese medicine crops. Therefore, on the basis of preliminary extraction of Chinese medicine crops, texture features 'Length/Width' are used to exclude roads with obvious vegetation characteristics. Length/Width is calculated as follow:

$$
\gamma=\frac{\mathbb{1}}{w}=\frac{a^{2}+\left((1-f) b^{2}\right)}{A}
$$

In the formula(3), a,b represent the length and width of the approximate boundary, and A represents the area of the object, indicating the weight.

When establishing a classification rule set, 150 spectral sample data of vegetation types uniformly distributed in the study area were selected based on the interpretation of multi-temporal remote sensing images and field survey data,including 50 samples of Chinese medicine crops ,50 samples of other crops and 50 samples of trees.

A total of 100 sample data of Chinese medicine crops and road texture uniformly distributed in the study area were selected, 
including 50 samples of texture features of Chinese medicine crops and 50 samples of road texture features. Calculating the maximum, minimum, and mean values of the sample (as shown in Table 4 and Table 5) and obtained the feature value chart for interpreting the target (Figure 2-4 to Figure 2-6).

Figure 5 shows that in the image of June 18th, the NDVI values of Chinese medicine crops are obviously higher than that of other crops, and the NDVI values of trees are similar to the medicine crops. At this time, the NDVI value can be used to better distinguish Chinese medicine crops and other crops. Figure 6 shows that in the image of June 18th, the Brightness values of Chinese medicine crops were significantly higher than the trees, while the Brightness values of other crops were partially cross-ranged with the Chinese medicine crops. At this time, the Brightness value can be used to distinguish between Chinese medicine crops and other crops. Therefore, the use of a combination of NDVI values and Brightness values can better distinguish between Chinese medicine crops, trees and other crops. As shown in Figure 7, most of the road's Length/Width value is significantly higher than that of Chinese medicine crops. Therefore, setting an appropriate aspect ratio value can eliminate most of the road's impact on information extraction results.

\begin{tabular}{ccccc}
\hline Feature & value & $\begin{array}{c}\text { Chinese } \\
\text { medicine crops }\end{array}$ & other crops & trees \\
\hline NDVI & $\min$ & 0.23 & 0.03 & 0.29 \\
& $\max$ & 0.55 & 0.20 & 0.49 \\
& $\operatorname{mean}$ & 0.50 & 0.13 & 0.39 \\
\hline Brightness & $\min$ & 448.83 & 480.65 & 370.20 \\
& $\max$ & 530.60 & 606.50 & 439.39 \\
& mean & 533.77 & 537.94 & 402.7 \\
\hline
\end{tabular}

Table4. Sample texture feature values of different ground objects.

\begin{tabular}{cccc}
\hline Feature & value & $\begin{array}{c}\text { Chinese medicine } \\
\text { crops }\end{array}$ & roads \\
\hline \multirow{2}{*}{ Length/Width } & $\min$ & 1.0 & 3.41 \\
\cline { 2 - 4 } & $\max$ & 12.0 & 19.50 \\
\cline { 2 - 4 } & mean & 3.02 & 8.79 \\
\hline
\end{tabular}

Table5. Spectral characteristic values of different types of ground objects.

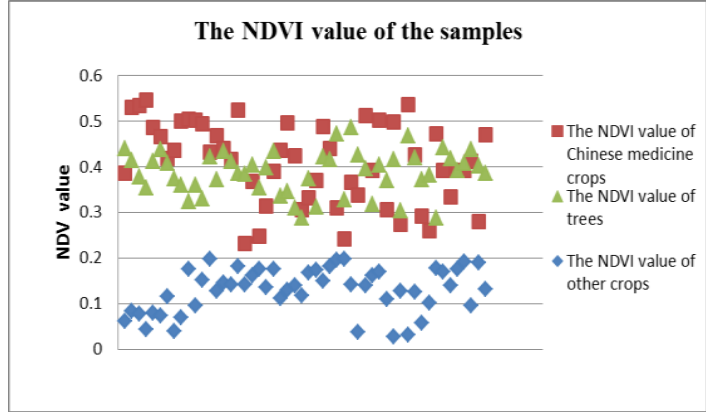

Figure 5. The NDVI value of the samples

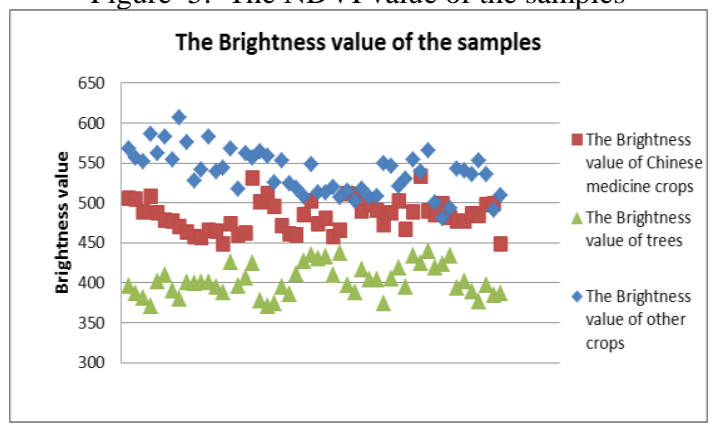

Figure 6.The Brightness value of the samples

cultivated area in the study area. As can be seen from Fig. 8, the planting areas of Chinese medicine crops in Qiaocheng District are mainly distributed in the central and northern regions,

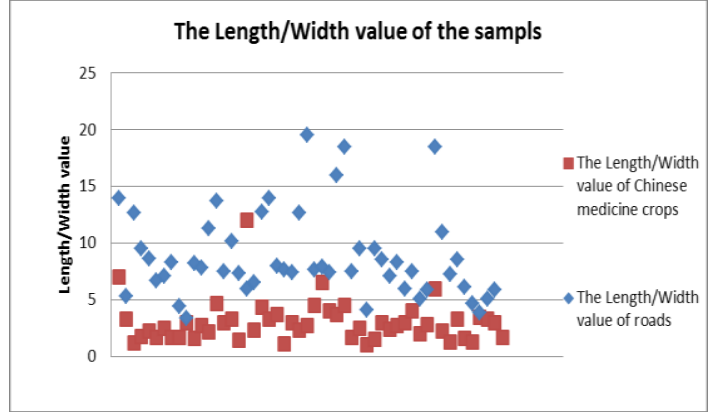

Figure 7. The Length/Width value of the samples

According to the above spectral characteristics and texture features, NDVI was selected to be greater than or equal to 0.25 and the Length/Width is less than 7 for the extraction of traditional Chinese medicine crops information.

\section{RESULTS AND ANALYSIS}

\subsection{Information extraction results}

The results of the extraction of Chinese medicine crops information in the study area are shown in Figure 8. The area of Chinese medicine planted in 2016 was estimated to be approximately $983,000 \mathrm{mu}$, accounting for $49.6 \%$ of the especially in townships such as Shijiuli, Shibali, Weigang, Gujing and Niuji. 


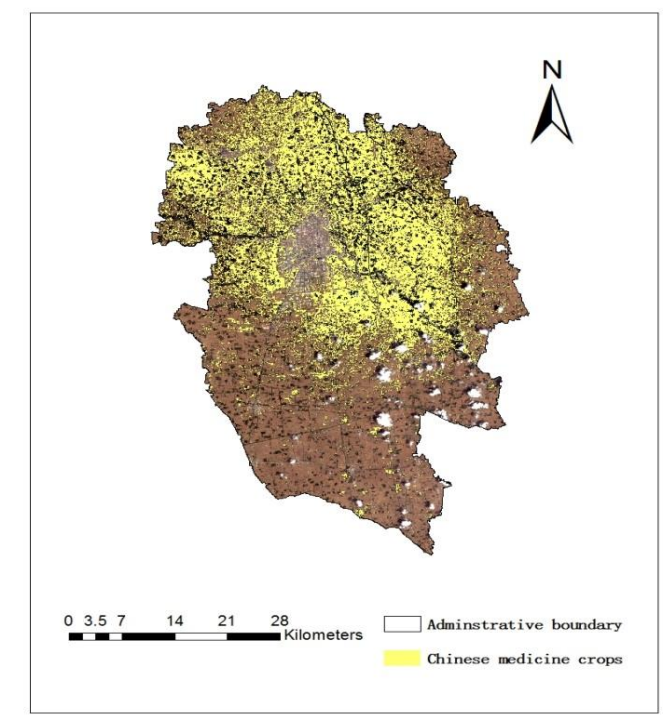

Figure 8. Spatial distribution of Chinese medicine crops in Qiaocheng District

\subsection{Accuracy evaluation}

In order to verify the results of the study, 544 block level field survey samples of Chinese medicine crops(test data) were used to construct an error matrix, that is, to verify the type of crop in the data and verify the location of each pixel in the data. Calculating the overall accuracy, producer accuracy, and user accuracy of the extraction results and quantitatively express the accuracy of the spatial distribution of the Chinese medicine crops. Accuracy evaluation results are shown in Table 6.The overall precision reached $93.56 \%$, of which the precision of Chinese medicine crops was $93.15 \%$, the accuracy of users was $98.47 \%$, and the precision was high.

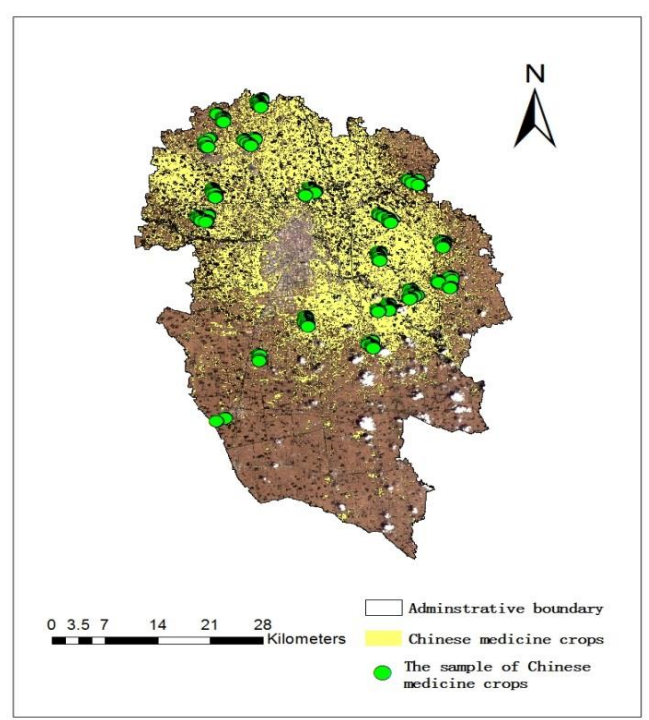

Figure 9. Spatial distribution of samples of Chinese medicine crops in Qiaocheng District.

\begin{tabular}{|c|c|c|c|c|}
\hline Classification data & $\begin{array}{c}\text { Chinese medicine } \\
\text { crops }\end{array}$ & Other features & Total & mapping accuracy \\
\hline $\begin{array}{l}\text { Chinese medicine } \\
\text { crops }\end{array}$ & 6848 & 289 & 7137 & $93.15 \%$ \\
\hline Other features & 103 & 1947 & 2050 & $94.98 \%$ \\
\hline Total & 6751 & 2236 & 9187 & \\
\hline User accuracy & $98.47 \%$ & $87.08 \%$ & & \\
\hline Overall accuracy & & & & \\
\hline
\end{tabular}

Table 6. Accuracy Evaluation of Chinese medicine crops 


\section{3 results analysis}

According to the statistics of Qiaocheng district in Bozhou city, the planting area of Chinese medicine crops is more than one million mu, which is consistent with the area extracted from this study. The cultivation area of Chinese medicinal materials is mainly composed of Shijiuli, Shibali, Weigang, Niuji,etc., mainly because these regions have unique geographical location advantages and resource advantages. Geographically, these areas are flat, rich in soil, mild in climate and moderate in rainfall. The soil is either sandy or sandy silt, which is suitable for the growth of Chinese medicine crops.There are larger Chinese medicinal materials markets in these regions, the transportation is convenient, attracting businessmen investment and convenient herbalist sale of Chinese medicinal materials, so the Chinese medicine crops are concentrated.

According to the actual field sampling points in the field, the accuracy of the Chinese medicine crops in Qiaocheng District was tested. The overall accuracy reached $93.56 \%$. The accuracy of the mapping reached $93.15 \%$ and the accuracy of the user reached $98.47 \%$, show that there are few missing and wrong points in the extraction process of Chinese medicine information, and the classification accuracy is high., this paper research method is feasible. Mainly reflected in: This paper is based on multi-temporal remote sensing imagery and objectoriented technology, using remote sensing image cognition and geography understanding as the visual angle, to interpret the image multi-scale segmentation, breaking through the traditional pixel-based classification method too Focusing on the local details of the feature and the difficulty of extracting the information on the overall structure of the feature, it ensures the integrity of the extraction plot and effectively eliminates the influence of the "pepper and salt phenomenon" on the extraction results. On this basis, the research fully exploits the characteristics of spectral features, texture features, etc. of Chinese medicine targets, and selects sample points to comprehensively analyze the optimal feature combinations so as to achieve accurate extraction of target objects.

\section{CONCLUSION AND DISCUSSION}

Generally speaking, this research method can accurately extract the planting area of Chinese medicine crops in Qiaocheng District, making the remote sensing method has a new breakthrough in the extraction of information on regional characteristics of crops. The research method has good application, promotion and practical value. At the same time, there are some deficiencies in the study, and we need to further strengthen the research in the following aspects:

(1) Due to the large number of varieties of Chinese medicine crops planted in the study area of this article, there are many phenomena between Chinese medicine crops. Although the general phenology period of the Chinese medicine crops in the region is the same, it does not exclude the difference in the phenological period of the individual varieties and it has a certain influence on the accuracy of the extraction of Chinese medicine crops. In the future research, it is necessary to strengthen research on the interpretation of various types of Chinese medicine crops.

(2) Due to the complexity of the remote sensing image itself, the phenomenon of " same object different spectrum, different object same spectrum " exists. Due to the fragmentation of the crop planting areas in some parts of the study area, even in the vigorous growth image, the spectral and texture characteristics are close to those of some vegetation roads, which has a certain influence on the accuracy of the extraction. In future studies, research needs to be strengthened in the establishment of multiple types of rule sets.

(3) According to related reports, with the increasing economic benefits brought to farmers by Chinese medicine crops,the planting area of Chinese medicine crops in Qiaocheng District has been increasing year by year, while the planting area of winter wheat and other food crops has shown a downward trend. This study only monitored the planting area of Chinese medicine crops in Qiaocheng District in 2016. The monitoring of changes in the cultivation of Chinese medicine crops in Qiaocheng District require further research.

The use of remote sensing methods has broken the traditional Chinese medicine crops survey methods and provided planners and decision makers with a new, more scientific and objective means. It will help the relevant departments fully understand the relevant information, correctly guide the farmers to adjust the agricultural planting structure, provide relevant technical guidance, and continuously improve the living standards of China's rural households.

\section{ACKNOWLEDGEMENTS}

This research was supported by the National Key R\&D Program of China (No.2017YFB0503903).

\section{REFERENCES}

Chen, S. S., Liu, Q. H., Chen, L. F., 2005. Research progress on remote sensing monitoring of sowing acreage of grain crops, Transactions of the Chinese Society of Agricultural Engineering, 21(6), pp.166-171.

Zhou, Q. B., 2004. The Situation and development of remote sensing of agricultural situation at home and abroad, Journal of China Agricultural Resources and Regional Planning, 25(5), pp.9-14.

Guo, L. P., Huang L. Q., Jiang, Y. X., 2005.Application of "3S" technology in sustainable utilization of traditional Chinese medicine resources, China Journal of Chinese Materia Medica, 30(18), pp.1397.

Chen, S. L.,Zhang B. G.,Yang Z., 2005. Design of national traditional Chinese medicine resource survey. China Journal of Chinese Materia Medica, 30(16), pp.1229.

Sun, Y. Z.,Guo L. P., Zhu W. Q., 2007.Remote sensing detection method for medicinal plant resources of different ecological environment types. China Journal of Chinese Materia Medica, 32(14), pp.1490.

Pan, H. J., Mao H, Peng W. F., 2014.Estimating Chinese Azeri medicine Pang-peng cultivation area Based on Remote Sensing and GIS technology-A Case Study of Lenglan Town and Xinglong Town in Luding County. China Journal of Chinese Materia Medica, 39(16), pp.3018-3022.

Zhang, X. B., Sun,Y. Z., Huang L. Q., 2009.Establishment of remote sensing monitoring methods and standards for wild rare species medicinal plants, China Journal of Chinese Materia Medica,34(13), pp.1741. 